19

\title{
The Round-Robin Service Discipline in Discrete Time for Phase-Type Distributed Packet-Lengths
}

\section{K. Laevens}

SMACS Research Group, University of Ghent

Sint-Pietersnieuwstraat $41, B-9000$ Ghent, Belgium.

Tel.: +32 926489 02, Fax.: +32926442 95, e-mail: kl@telin.rug.ac.be

\begin{abstract}
The buffer contents in a discrete-time single-server queue is analyzed under a round-robin service discipline. This service discipline is related to the continuous-time processor-sharing discipline and models the operation of certain types of input-buffered ATM-switches when variable-length packets are routed. The packet-length distribution is arbitrary here but of phase-type. Through a generating-functions approach, a set of functional equations is derived from which a straightforward algorithm to calculate the mean buffer contents is obtained. Numerical examples illustrate the main characteristics of the round-robin service discipline and its relation to the first-come-first-serve service discipline. Hereby, implications for the performance of the input-buffered ATM-switches are given special attention.
\end{abstract}

\section{Keywords}

Round-Robin Service Discipline, Discrete-Time, Phase-Type Distributions, Generating Functions

Performance and Management of Complex Communication Networks

T. Hasegawa, H. Takagi \& Y. Takahashi (Eds.) (C) 1998 IFIP. Published by Chapman \& Hall 


\section{INTRODUCTION}

For some time now, the round-robin (RR) service discipline, in both continuous time (Lam et al. 1981, Muntz 1972) and discrete time (Daduna et al. 1981, Schassberger 1981), and the related continuous-time processor-sharing (PS) discipline (Kleinrock 1976, Schassberger 1984, van den Berg et al. 1991) have been studied extensively, mainly in the context of multi-tasking or time sharing in computer systems (Jaiswal 1982). Recently, however, the same disciplines have received renewed attention in the context of ATM-switching, for reasons to be explained next.

In input-buffered ATM-switches (Awdeh et al. 1995, Karol et al. 1987), cells at the head-of-line (HOL) positions of the input queues compete for routing with cells at the HOL positions of other input queues having the same destination or output address. This process has been succesfully modelled by means of virtual queues (Karol et al. 1987). For uncorrelated cell destinations, results for (a network of) continuous-time $M / D / 1$ or discrete-time GI-D-1 queues can be used to assess the performance. Typical performance measures include maximum throughput and mean system contents. For correlated cell destinations, this approach remains valid, but more complicated queue models have to be developed, as e.g. in (Cao 1995, Cao et al. 1995, Jacob et al. 1995, Laevens et al. 1996a, Li 1992). Usually the correlation is modelled by packets, consisting of a variable number of cells having the same destination. Important is that when in this case the switch selection policy is such that cells are served on a first-come-first-served (FCFS) basis inside the virtual queues, a RR discipline for HOL packets results. For a more detailed discussion of these issues, we refer to the cited papers.

Our analysis mainly focuses on performance measures on packet level. It requires, however, minor effort to reformulate things on cell level. At this level, the system behaves as a single-server cell-buffer with a FCFS discipline among cells, whereby a form of feedback is present between cell-departure and cellarrival process. This feedback introduces correlation between waiting times of different cells belonging to a same packet. Results on feedback queues can be found in e.g. (Disney et al. 1984, Lam et al. 1981, Schassberger 1984, Takács 1963, van den Berg et al. 1989, van den Berg et al. 1991).

The structure of the paper is as follows. In section 2, the mathematical model is defined. Phase-type distributions are introduced for the packet lengths and the arrival process is looked at in some detail. Section 3 , where the per-type buffer contents is analyzed based on a generating-functions approach, makes up the main part of this paper. A system of functional equations is derived, in subsection 3.1 , from which mean values are extracted in subsection 3.2. Two numerical examples given in subsection 3.3, highlight the main characteristics of the RR discipline and its implications for switch performance. Hereby, results for the better known FCFS discipline are used as reference. Waiting times are addressed in section 4 and conclusions drawn in section 5 . 


\section{THE MATHEMATICAL MODEL}

\subsection{The buffer}

The buffer analyzed here, has a single server and an infinite waiting room. It is fed by packets (or jobs, customers, ...) consisting of a variable number of fixed-length cells. It operates in a time-slotted fashion, i.e., time is divided into consecutive intervals of unit length, called slots, during which a single cell can be served. The time to transmit (or route, serve, ...) a cell will be chosen as time unit.

The numbers of cells within each packet, the so-called packet lengths, are independent and identically distributed (iid) random variables (rv's) with a common distribution $s(n)=\operatorname{Prob}[s=n](n=1,2, \ldots)$. Hereby, $s$ denotes the length of a generic packet. The probability generating function (pgf) associated with this distribution, is defined as

$S(z)=\mathrm{E}\left[z^{s}\right]=\sum_{n=1}^{+\infty} s(n) z^{n}$

The mean packet length is then given by

$\mathbf{E}[s]=S^{\prime}(1)=\sum_{n=1}^{+\infty} s(n) n$

In this paper, we will assume that packet lengths follow a phase-type distribution, as to be discussed in subsection 2.3.

As for the arrival process, we will assume that it constitutes a so-called GI arrival process (Bruneel et al. 1993). If we denote by $a_{k}$ the number of newly arriving packets during slots $k(k=\ldots, 0,1, \ldots)$, we assume that the $a_{k}$ are iid rv's. Their common distribution will be denoted as $a(n)=\operatorname{Prob}\left[a_{k}=n\right]$ and the associated pgf as

$A(z)=\mathrm{E}\left[z^{a_{k}}\right]=\sum_{n=0}^{+\infty} a(n) z^{n}$.

The mean number of arrivals per slot is given by $\lambda=\mathrm{E}\left[a_{k}\right]=A^{\prime}(1)$. For reasons of stability, $\lambda$ should be such that the load $\rho=\lambda \mathrm{E}[s]$ is less than unity. 


\subsection{The round-robin service discipline}

Under queueing disciplines such as FCFS or LCFS (last-come-first-served), packets are considered indivisible units and are served uninterruptedly once they access the server. No relation exists between their actual length and the waiting time they experience, which is a form of unfairness (Kleinrock 1976). To overcome this, other service disciplines have been devised in the past, one of which is the so-called round-robin (RR) service discipline. Under this discipline, packets circulate in a FCFS queue, whereby the packet at the headof-the-line (HOL) position is granted a single slot service time, i.e., a single cell of the packet is transmitted. If afterwards the packet still needs servicing, it has to rejoin the queue at the rear. As such, the RR discipline is the discretetime analog of the processor-sharing (PS) discipline (Kleinrock 1976, van den Berg et al. 1991), for it distributes the available service (or channel, switching, ...) capacity in a more or less uniform way over all packets present in the system.

Due to the time-slotted operation of the system, an issue concerning simultaneity arises, not found in continuous-time models. A packet may rejoin the queue during a slot in which also new packets arrive, and these packets can mix with eachother in a number of ways. Here, we will assume that newly arriving packets get queued ahead of a packet rejoining the queue. This seems to be the most natural choice for systems whereby the arrival process is not synchronized with the service process, so that packets can arrive at any point in time during a slot, while a packet can only rejoin the queue after receiving service, i.e., at the end of a slot. Another option is to assume that no distinction can be made between newly arriving packets and the packet rejoining the queue. This seems to be more likely within the context of input-buffered ATM-switches, since the queues within that context are only logical queues (Karol et al. 1987). The analysis presented here, needs only to be modified at some minor points to account for this. However, doing so does complicate the resulting expressions to some extent, and, as such, could obscure the main ideas of the analysis. More detailed research has shown that the exact way in which packets mix has only a minor effect on performance measures of interest.

\subsection{Phase-type distributed packet lengths}

In (Laevens et al. 1996a), the above described queue with RR discipline was analyzed under the assumption of geometrically distributed packet lengths, i.e., for $s(n)=\alpha(1-\alpha)^{n-1}$. A key characteristic of the geometric distribution is its memoryless property, i.e., the fact that $P[s=n \mid s \geq n]=\alpha$ does not depend on $n$. This simplified the analysis in (Laevens et al. 1996a) considerably, since no information about the service already received was needed to 
determine whether or not a packet would leave the system after receiving a slot of service. The main idea of this paper is to extend that analysis to more general forms of distributions, while at the same time trying to preserve some sort of memoryless property. Discrete phase-type distributions, while being fairly general, allow just that.

Define $s$ as the number of transitions until absorption in a finite state Markov chain with $N$ non-absorbing states and a single absorbing state. Further, denote the $k$ th state visited by the Markov chain as $\varphi_{k}$. The distribution of $s$ is then fully specified by the probabilities $m(i \mid j)(i, j=1, \ldots, N)$ of a transition from non-absorbing state $j$ to non-absorbing state $i$, and the probabilities $\nu_{j}=\operatorname{Prob}\left[\varphi_{1}=j\right](j=1, \ldots, N)$ of the Markov chain starting in the non-absorbing state $j$. The probabilities $\tau_{j}(j=1, \ldots, N)$ of a transition from non-absorbing state $j$ to the absorbing state are given by $\tau_{j}=1-\sum_{i=1}^{N} m(i \mid j)$. For the remainder of the paper, we will use vectors and matrices to reduce notational overhead. Therefore, let us define the following matrix and rowvectors: $[\mathbf{M}]_{i j}=m(i \mid j),\langle\mathbf{v}\rangle_{i}=\nu_{i},\langle\mathbf{T}\rangle_{i}=\tau_{i}(i, j=1, \ldots, N)$, and $\mathbf{e}=\langle 1, \ldots, 1\rangle$.

The distribution associated with $s$ can be determined by observing that

$\operatorname{Prob}[s=n]=\sum_{j=1}^{N} \operatorname{Prob}\left[s=n \mid \varphi_{1}=j\right] \nu_{j}=\operatorname{Prob}\left[s=n \mid \phi_{1}\right] \mathbf{v}^{t}$

Hereby, we introduced the notation $\left\langle\operatorname{Prob}\left[s=n \mid \phi_{1}\right]\right\rangle_{i}=\operatorname{Prob}\left[s=n \mid \varphi_{1}=i\right]$ and used $\mathbf{v}^{t}$ to denote the transpose of $\mathbf{v}$. Now, $\operatorname{Prob}\left[s=n+1 \mid \phi_{1}\right]=\operatorname{Prob}[s=$ $\left.n \mid \phi_{1}\right] \mathbf{M}$ and $\operatorname{Prob}\left[s=1 \mid \phi_{1}\right]=\mathbf{T}=\mathbf{e}(\mathbf{I}-\mathbf{M})$, whereby $\mathbf{I}$ is the $N \times N$ unit matrix. Hence, $\operatorname{Prob}\left[s=n \mid \phi_{1}\right]=\mathbf{e}(\mathbf{I}-\mathbf{M}) \mathbf{M}^{n-1}$ and $\operatorname{Prob}[s=n]=$ $\mathbf{e}(\mathbf{I}-\mathbf{M}) \mathbf{M}^{n-1} \mathbf{v}^{t}$. Introducing z-transforms, we obtain

$S\left(z \mid \phi_{1}\right)=\left\langle\mathrm{E}\left[z^{s} \mid \varphi_{1}=1\right], \ldots, \mathrm{E}\left[z^{*} \mid \varphi_{1}=N\right]\right\rangle=z \mathbf{e}(\mathbf{I}-\mathbf{M})(\mathbf{I}-z \mathbf{M})^{-1}$

for the pgf's of $s$ conditioned on the initial state $\varphi_{1}$, and

$S(z)=\mathrm{E}\left[z^{s}\right]=S\left(z \mid \phi_{1}\right) \mathbf{v}^{t}=z \mathbf{e}(\mathbf{I}-\mathbf{M})(\mathbf{I}-z \mathbf{M})^{-1} \mathbf{v}^{t}$

for the unconditional pgf. Derivatives of $S(z)$ are given by

$S^{(n)}(z)=n ! \mathbf{e}(\mathbf{I}-\mathbf{M})(\mathbf{I}-z \mathbf{M})^{-(n+1)} \mathbf{M}^{n-1} \mathbf{v}^{t}$

From this, the mean packet length $\mathrm{E}[s]$ follows as

$\mathrm{E}[s]=S^{\prime}(1)=\mathbf{e}(\mathbf{I}-\mathbf{M})^{-1} \mathbf{v}^{t}$

and the variance of the packet length $V[s]$ as $V[s]=\mathrm{E}\left[s^{2}\right]-\mathrm{E}[s]^{2}=S^{\prime \prime}(1)+$ $S^{\prime}(1)-S^{\prime}(1)^{2}$. 
It is not the purpose of this paper to go deeper into the properties of phasetype distributions. For more information, we refer the reader to (Neuts 1981) or (Asmussen et al. 1996). In the latter, the problem of fitting continuous phase-type distributions is addressed. Special cases of discrete phase-type dis-

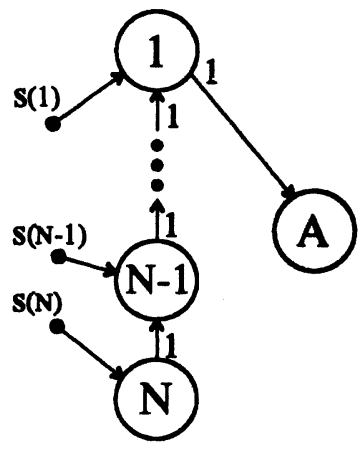

polynomial

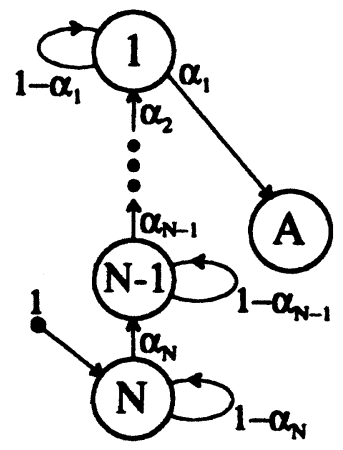

convolution of geometrics

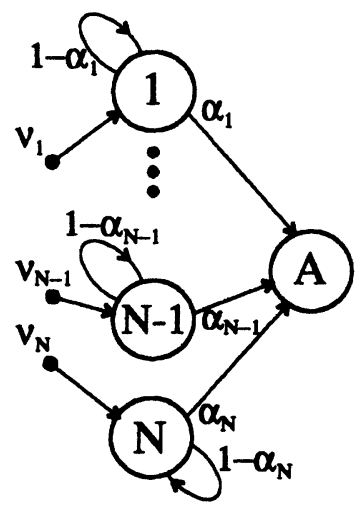

mixture of geometrics

Figure 1 Markov chains for three special cases of phase-type distributions tributions are, besides the geometric distribution, polynomial distributions, convolutions of geometrics and mixtures of geometrics. For these cases, the pgf of the packet length is given by

$\sum_{i=1}^{N} s(n) z^{n}, \quad \prod_{i=1}^{N} \frac{\alpha_{i} z}{1-\left(1-\alpha_{i}\right) z}$ and $\sum_{i=1}^{N} \nu_{i} \frac{\alpha_{i} z}{1-\left(1-\alpha_{i}\right) z}$

respectively. Corresponding Markov chains are depicted in Figure 1, from which the vector $\mathbf{v}$ and the matrix $\mathbf{M}$ are easily derived.

In what follows, we will call a packet being of type $j(j=1, \ldots, N)$ when the Markov chain associated with its service time is in state $j$. After a cell of that packet has been served, the packet then either changes from type $j$ to type $i$, with probability $m(i \mid j)$, or leaves the queue, with probability $\tau_{j}$. Its initial type is $j$ with probability $\nu_{j}$.

\subsection{A closer look at the arrival process}

The description in subsection 2.1 of the arrival process only involved the total number of arrivals during a given slot. In what follows, it will be easier to 
make use of the joint pgf of the per-type arrival process, i.e., of the numbers $a_{i, k}$ of type $i$ packets arriving during slot $k$. Therefore, define

$B(z)=B\left(z_{1}, \ldots, z_{N}\right)=\mathrm{E}\left[z_{1}^{a_{1, k}} \cdots z_{N}^{a_{N, k}}\right]$

Since every new packet is initially of type $i$ with probability $\nu_{i}$, we have

$B(z)=A\left(\nu_{1} z_{1}+\ldots+\nu_{N} z_{N}\right)=A\left(\mathbf{v} z^{t}\right)$.

Nothing, however, prevents us from specifying the joint generating function $B(z)$ in a more general way, using e.g. $N$ independent GI arrival processes, one for each type. (The load $\rho$ is then no longer given by $\lambda E[s]$, but by $\rho=\sum_{i=1}^{N} \lambda_{i} \mathrm{E}\left[s \mid \varphi_{1}=i\right]$, with $\lambda_{i}=\mathrm{E}\left[a_{i, k}\right]$.)

This approach results, generally speaking, in the position of an arbitrarily chosen or tagged packet within the bulk of new packets in which it arrives, to be dependent on the type of that tagged packet (Laevens et al. 1996b). This would further complicate our analysis, as will be discussed at the appropriate time. Therefore, we restrict ourselves to the case of a so-called probabilistic mix, whereby $B(z)$ is given as in equation (2).

The position of a tagged packet within the bulk of arrivals during the slot of its arrival, can be described by the vectors $\langle\mathbf{f}\rangle_{i}=f_{i}$ and $\langle\mathbf{g}\rangle_{i}=g_{i}$, whereby $f_{i}$ and $g_{i}$ are the numbers of type $i$ packets arriving during the same slot as the tagged packet and queued ahead respectively behind it at the end of that slot. For the arrival process considered here, the joint pgf of $\mathbf{f}$ and $g$ is of the form (Laevens et al. 1996b)

$F(x, y)=\mathrm{E}\left[x^{\mathbf{f}} y^{\mathrm{g}}\right]=\frac{A\left(\mathbf{v} x^{t}\right)-A\left(\mathbf{v} y^{t}\right)}{\lambda\left(\mathbf{v} x^{t}-\mathbf{v} y^{t}\right)}$

whereby we used the notation $x^{\mathbf{f}}=\prod_{i=1}^{N} x_{i}^{f_{1}}$ and $y^{\mathbf{g}}=\prod_{i=1}^{N} y_{i}^{g_{i}}$.

\section{ANALYSIS OF THE BUFFER CONTENTS}

\subsection{A generating-functions approach}

The analysis of the buffer contents should necessarily take into account the numbers of different types of packets in the system, i.e., the per-type buffer contents, since the type of a packet determines the probability of it leaving the system after receiving a slot of service. A slot-to-slot based analysis of this per-type buffer contents, in other words, the determination of the per-type buffer contents at the beginning of slot $k+1$ from that at the beginning of slot $k$, seems not feasible because it also requires knowledge of the exact order 
in which packets are queued. This information is needed to determine what type of packet will be the next to receive service. To overcome this problem, we rely on an analysis of the different cycles a tagged packet goes through while visiting the system.

Let us denote the vector of the numbers of type $i$ packets in the system at the beginning of slot $k$ by $\left\langle\mathbf{u}_{k}\right\rangle_{i}=u_{i, k}$. The joint pgf

$U(z)=\lim _{k \rightarrow+\infty} E\left[z^{u_{k}}\right]=\lim _{k \rightarrow+\infty} E\left[z_{1}^{u_{1, k}} \cdots z_{N}^{u_{N, k}}\right]$

of these numbers at the beginning of a slot in equilibrium, can be expressed as

$U(z)=p_{0}+\sum_{j=1}^{N} L_{j}(z) z_{j}=p_{0}+\mathbf{L}(z) z^{t}$.

The functions $\mathbf{L}(z)_{j}=L_{j}(z)$ in the above, are the partial joint pgf's of the numbers $\langle q\rangle_{i}=q_{i}$ of type $i$ packets in the queue, the buffer without the server, and the occurence of a type $j$ packet being in service, observed at the beginning of a slot, i.e.,

$L_{j}(z)=\mathrm{E}\left[z^{\mathrm{q}} \cdot I\left(\varphi_{*}=j\right)\right]$.

where $I(\cdot)$ is the indicator function and $\varphi_{\text {. }}$ the type of the packet in service. The probability $p_{0}$ is the probability of the system being empty and is given by $1-\rho$.

Next, we will analyze the different cycles a tagged packet goes through while in the buffer, whereby we assume the equilibrium situation prevails. A cycle starts at the end of a slot wherein the packet joins the queue at the rear, be it either by arriving to the queue for the first time or by rejoining the queue after receiving a slot of service. A cycle ends at the beginning of the slot in which the packet will receive service, i.e., after it has queued for some time and reached the HOL position. Note that the slot during which service is received is not included in a cycle. In order to keep track of how the queue contents changes during different cycles, we introduce the functions $Q_{n}(z)=\mathrm{E}\left[z^{q_{n}}\right]$ that are the joint pgf's of the per-type queue contents $\mathbf{q}_{n}$ observed by the tagged packet at the end of the $n$th cycle, i.e., just before it receives its $n$th slot of service. The following relation holds for $n \geq 1$

$Q_{n+1}(z)=B(B(z)(\mathbf{T}+z \mathbf{M})) Q_{n}(B(z)(\mathbf{T}+z \mathbf{M}))$.

It is based on the following observations. At the beginning of a cycle other than the first, the tagged packet will be behind all other packets in the system, due to assumptions we made in subsection 2.2 on how packets mix with each 
other. The per-type number of packets ahead of the tagged packet at that time, consists of contributions of packets already in the system when the tagged packet entered service, which results in the factor $Q_{n}(\cdot)$, and of the contribution of the packets that arrived during that slot of service, which results in the factor $B(\cdot)$. The arguments in the right-hand side of equation (6), originate from the following observations. Before the tagged packet can enter service, all other packets first have to receive a slot of service. During that time, the queue builds up behind the tagged packet, as follows. During each slot, one per packet, new packets can arrive, as specified by the joint $\operatorname{pgf} B(z)$ of the GI arrival process. Besides these packets, packets rejoining the queue also contribute. A packet of type $j$ will rejoin the queue as a packet of type $i$ with probability $m(i \mid j)$, or it will leave the queue, thus not contributing to the buildup of the queue, with probability $\tau_{j}$. For each packet of type $j$, a total contribution is thus made, described by $B(z)\left(\tau_{j}+m(1 \mid j) z_{1}+\ldots+m(N \mid j) z_{N}\right)$. In vector notation, this results in the arguments $B(z)(\mathbf{T}+z \mathbf{M})$ in the righthand side of the above equation.

The first cycle differs somewhat from the others, since it starts with the tagged packet joining the queue as a new arrival. At that moment, the tagged packet is not necessarily the last packet in the queue, unlike at the beginning of the other cycles. This is not only due to our assumption about how packets rejoin the queue, but also due to the random way in which the packet was chosen, see subsection 2.4. The per-type numbers of packets other than the tagged packet, in the system at the start of the first cycle, have joint pgf

$P(\boldsymbol{x}, \boldsymbol{y})=F(\boldsymbol{x}, \boldsymbol{y})\left(p_{0}+\mathbf{L}(\boldsymbol{x})(\mathbf{T}+\boldsymbol{y} \mathbf{M})^{t}\right)$,

whereby $x$ refers to the packets ahead of the tagged packet, and $y$ to those behind it. The term $F(x, y)$ should be obvious in light of subsection 2.4. The other terms result from the way in which the system contents in equilibrium was decomposed, see equation (4). Packets in the queue, i.e., not in service, at the beginning of the slot of arrival of the tagged packet, will still be in the system at the end of that slot. They will necessarily be ahead of the tagged packet, hence the term $\mathbf{L}(x)$. The packet in service during the slot of arrival of the tagged packet, if any, may result in a packet rejoining the queue at the end of that slot, possibly with a change of type, as explained above. This yields the term $(\mathbf{T}+\boldsymbol{y} \mathbf{M})$, in which $\boldsymbol{y}$ appears, since a rejoining packet will be queued behind the tagged packet. The per type queue contents at the end of the first cycle can now be related to this initial situation as

$$
\begin{aligned}
Q_{1}(z) & =P(B(z)(\mathbf{T}+z \mathbf{M}), z) \\
& =F(B(z)(\mathbf{T}+z \mathbf{M}), z)\left(p_{0}+\mathbf{L}(B(z)(\mathbf{T}+z \mathbf{M}))(\mathbf{T}+z \mathbf{M})^{t}\right) .
\end{aligned}
$$

Packets ahead of the tagged packet at the beginning of the first cycle, related to argument $x$ of $P(x, y)$, result in new arrivals and packets rejoining the 
queue, in the same way as those ahead of the packet at the beginning of other cycles. Packets behind the tagged packet at the beginning of the first cycle, argument $y$ of $P(x, y)$, will still be behind it at the end of the cycle. These observations readily yield the above equation.

Note that the type of the tagged packet was of no importance so far. Equation (6) is valid for any tagged packet, which implies that if $Q_{n}(z)$ is independent of the initial type of the tagged packet, so is $Q_{n+1}(z)$, or, if $Q_{1}(z)$ is, then all $Q_{n}(z)$ are. Now, $Q_{1}(z)$ is independent of the initial type of the tagged packet, because of the special nature of the per-type arrival process, as argued in subsection 2.4 .

The partial pgf's $L_{j}(z)$, equation (5), can be expressed as

$L_{j}(z)=\lambda \sum_{n=1}^{+\infty} Q_{n}(z) \operatorname{Prob}\left[(s \geq n) \cdot\left(\varphi_{n}=j\right)\right]$.

Every packet that enters the system, $\lambda$ per slot on average, contributes to the probability of finding a type $\boldsymbol{j}$ packet in service, whenever its service time is at least $n$ slots and $\varphi_{n}=j$. At that time, the queue contents observed by that packet, is described by $Q_{n}(z)$. Taking into account all possible values of $n$, we easily obtain the above. Note once more that the special nature of the arrival process allowed us to disregard the initial type of packets. Similarly as in subsection 2.3 , it is possible to derive the result

$\operatorname{Prob}\left[(s \geq n+1) \cdot\left(\varphi_{n}=j\right)\right]=\sum_{k=1}^{N} \operatorname{Prob}\left[(s \geq n) \cdot\left(\varphi_{n-1}=k\right)\right] m(j \mid k)$

for $n \geq 1$ and $\operatorname{Prob}\left[(s \geq 1) \cdot\left(\varphi_{n}=j\right)\right]=\nu_{j}$. Using these relations, one obtains from equations $(9)$ and $(6)$ that

$$
L_{j}(z)=\lambda Q_{1}(z) \nu_{j}+B(B(z)(\mathbf{T}+z \mathbf{M})) \sum_{k=1}^{N} L_{k}(B(z)(\mathbf{T}+z \mathbf{M})) m(j \mid k)
$$

or, in vector notation,

$\mathbf{L}(z)=\lambda Q_{1}(z) \mathbf{v}+B(B(z)(\mathbf{T}+z \mathbf{M})) \mathbf{L}(B(z)(\mathbf{T}+z \mathbf{M})) \mathbf{M}^{t}$.

Combining this with equation (8), we finally obtain

$$
\begin{aligned}
\mathbf{L}(z)= & \lambda F(B(z)(\mathbf{T}+z \mathbf{M}), z)\left(p_{0}+\mathbf{L}(B(z)(\mathbf{T}+z \mathbf{M}))(\mathbf{T}+z \mathbf{M})^{t}\right) \mathbf{v} \\
& +B(B(z)(\mathbf{T}+z \mathbf{M})) \mathbf{L}(B(z)(\mathbf{T}+z \mathbf{M})) \mathbf{M}^{t}
\end{aligned}
$$

This equation represents a system of $N$ coupled functional equations for the 
functions $L_{i}(z)(i=1, \ldots, N)$. To solve it for these functions would be a formidable, if not impossible, task. However, even as it stands, we can extract useful performance measures from it, as will be shown next.

\subsection{Derivation of the first moments}

Putting $z=\mathbf{e}$ in equation (10) and using the normalization condition $U(\mathbf{e})=$ 1 , we obtain $\mathbf{L}(\mathbf{e})=\lambda \mathbf{v}+\mathbf{L}(\mathbf{e}) \mathbf{M}^{t}$ or $\mathbf{L}(\mathbf{e})=\lambda \mathbf{v}\left(\mathbf{I}-\mathbf{M}^{t}\right)^{-1}$. The vector $\mathbf{L}(\mathbf{e})$ contains the probabilities of finding a type $i(i=1, \ldots, N)$ packet in service at the beginning of an arbitrary slot in regime.

Taking partial derivatives of both sides of equation (10), and performing some straightforward but tedious algebra, one finds for the matrix $\mathbf{K}$, defined as

$$
[\mathbf{K}]_{i j}=\left.\frac{\partial L_{i}(\boldsymbol{z})}{\partial z_{j}}\right|_{\boldsymbol{z}=\mathbf{e}},
$$

the following system of $N^{2}$ linear equations

$$
\mathbf{K}=\mathbf{D} \mathbf{K} \mathbf{D}^{t}+\mathbf{E}
$$

whereby the matrices $\mathbf{D}$ and $\mathbf{E}$ are given by

$\mathbf{D}=\lambda \mathbf{e}^{t} \mathbf{v}+\mathbf{M}^{t}$

and

$\mathbf{E}=\frac{A^{\prime \prime}(1)}{2} \mathbf{v}^{t} \mathbf{v}(\mathbf{I}+\mathbf{D})+\lambda\left(\mathbf{v}^{t} \mathbf{L}(\mathbf{e}) \mathbf{M}^{t}+\mathbf{M} \mathbf{L}^{t}(\mathbf{e}) \mathbf{v D}\right)$

respectively. Once $\mathbf{L}(\mathbf{e})$ and $\mathbf{K}$ have been obtained, the mean per-type buffer contents can be calculated from $\mathrm{E}[\mathbf{u}]=\left\langle\mathrm{E}\left[u_{1}\right], \ldots, \mathrm{E}\left[u_{N}\right]\right\rangle=\mathbf{L}(\mathbf{e})+\mathbf{e K}$. The mean buffer contents then follows as $\mathbf{E}[u]=E[u] \mathbf{e}^{t}$.

Equation (11) represents a system of $N^{2}$ linear equations, whereby a $N^{2} \times$ $N^{2}$ matrix is thus involved. For small $N$, say up to values of 30 , this causes no special problems, and the equations can be solved quite easily with e.g. the Gauss-Seidel method. For larger $N$, the matrix involved may become too large, especially since, in general, it has no special structure nor is it sparse. Repeated application of equation (11) yields

$$
\mathbf{K}=\sum_{n=0}^{+\infty}(\mathbf{D})^{n} \mathbf{E}\left(\mathbf{D}^{t}\right)^{n}
$$


Here, the matrices involved are of order $N \times N$ only. We did not investigate yet whether or not this approach is feasible, i.e., if it convergences fast enough and if it is numerically stable. Still other solution methods can be thought of, as e.g. diagonalization of $\mathbf{D}$. Suppose $\mathbf{D}=\mathbf{N}^{-1} \mathbf{G N}$, whereby $\mathbf{G}$ is diagonal, with $[G]_{i i}=\gamma_{i}$. Then, for the matrix $\mathbf{K}^{*}=\mathbf{N K N}^{t}$, we obtain from equation (11) that $\mathbf{K}^{*}=\mathbf{G K}^{*} \mathbf{G}+\mathbf{E}^{*}$ with $\mathbf{E}^{*}=\mathbf{N E N ^ { t }}$. From this we get

$\left[\mathbf{K}^{*}\right]_{i j}=\frac{\left[\mathbf{E}^{*}\right]_{i j}}{1-\gamma_{i} \gamma_{j}}$

for $i, j=1, \ldots, N$, and, finally, $\mathbf{K}=\mathbf{N}^{-1} \mathbf{K}^{*}\left(\mathbf{N}^{-1}\right)^{t}$. For some special cases of the packet-length distribution, explicit expressions for the matrices $\mathbf{N}$ and $\mathbf{G}$ can be derived. For example, for polynomial distributions which typically require a large number $N$ of states in the underlying Markov chain, one can show that $\gamma_{i}^{-1}$ is a solution of the polynomial equation

$1=\rho x \frac{S(x)-1}{S^{\prime}(1)(x-1)}$.

The function on the right-hand side of this equation equals the pgf of a residual lifetime, if one considers a discrete-time renewal process with renewal periods having pgf $S(x)$. This elegant structure rises the suspicion that for this special case, or even in general, further analysis of equation (11) could lead to an explicit expression for the matrix $\mathbf{K}$ or for the mean per-type or total buffer contents, $\mathbf{E}[\mathbf{u}]$ and $E[u]$ respectively.

With this remark, we end the analytical part of the paper and turn to some numerical examples to study the characteristics of the RR discipline.

\subsection{Numerical examples}

\section{(a) Example 1}

In order to analyze the effect of the packet-length distribution on the buffer contents, we consider, besides the geometric $(G)$ distribution, three other cases: deterministic (D) (as a special case of a polynomial distribution), a convolution of geometrics $(C)$ and a mixture of geometrics $(M)$. We refer again to Figure 1 for the associated Markov chains. The mean packet length is 10.0 in all cases. The pgf for case D equals $S(z)=z^{N}$, with $N=10$. Parameters for the other cases are as follows. For case $\mathrm{C}$, the number of geometrics convolved is $N=2$ whereby $\alpha_{1}=\alpha_{2}$. For case $M$, the number of geometrics mixed is $N=2$. The weights are $\nu_{1}=\nu_{2}=0.5$ and the mean values $1 / \alpha_{1}=5.0$ and $1 / \alpha_{2}=15.0$. The variances of the resulting distributions equal $0.0,40.0$, 90.0 and 140.0 for cases $D, C, G$ and $M$ respectively. The arrival process was assumed to be Poisson, i.e., $A(z)=e^{\lambda(z-1)}$. 


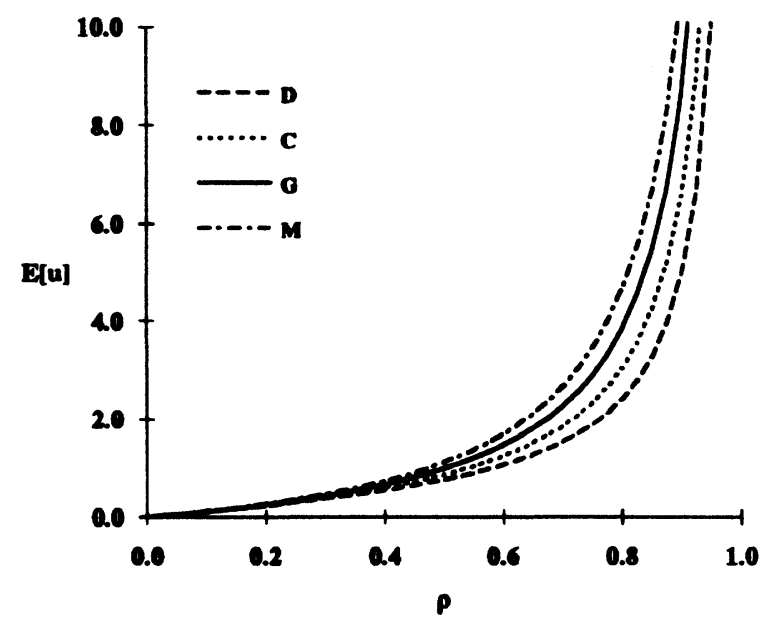

Figure 2 Mean buffer contents (FCFS discipline)

In Figure 2, the mean buffer contents is shown as a function of the load. It only shows results for a FCFS discipline. Curves for the RR discipline were not displayed, since these curves coincide (case G) or nearly coincide (cases $D, C$ and $M$ ) with the curve for case $G$ under the FCFS discipline, as to be discussed next. For a Poisson arrival process, the mean buffer contents under a FCFS discipline is given by (Bruneel et al. 1993)

$\mathrm{E}[u]=\rho+\frac{\lambda^{2}\left(\mathrm{E}[s]^{2}+V[s]\right)}{2(1-\rho)}$,

and, as clearly shows in Figure 2, an increasing packet-length variance results in an increasing mean buffer contents, especially when the load is high.

For the RR discipline, we plotted in Figure 3, as a function of the load, the ratio $\Delta$ of the mean buffer contents, given a certain packet-length distribution, to the mean buffer contents for a geometric packet-length distribution with same mean, i.e., we plotted the quantity

$\Delta=\mathrm{E}[u]_{(\star)} / \mathrm{E}[u]_{(\mathrm{G})}$.

The figure shows that the variance of the packet-length distribution is of only minor influence, as far as the mean buffer contents is concerned. For low loads, packet-length variances smaller than that for a geometric distribution, cases D and C, lead to a slightly larger mean buffer contents, while for high loads, they lead to a slightly smaller one. For larger packet-length variances than that for a geometric distribution, case $M$, the opposite holds. The discretetime RR discpline thus behaves nearly as the continuous-time PS discipline, 


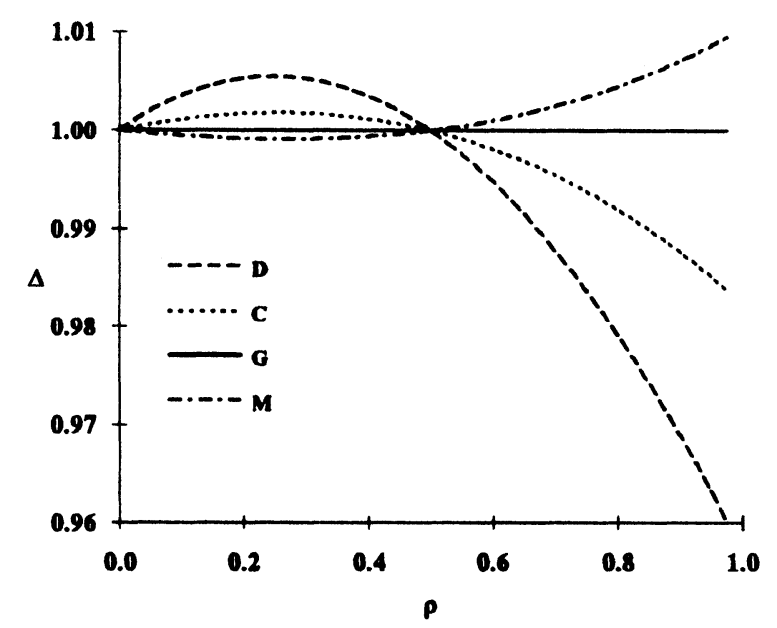

Figure 3 Ratio of mean buffer contents (RR discipline)

under which the mean buffer contents is influenced by the mean packet-length only (Kleinrock 1976). The fact that, for a Poisson arrival process, all cases yield a same mean buffer contents at load $\rho=0.5$, does not seem to have an obvious intuitive explanation.

Comparing the FCFS and RR disciplines, we already mentioned that they yield the same mean buffer contents when packet lengths are geometrically distributed. In light of the system equation for that buffer contents, which, for this special case, is of the form

$u_{k+1}= \begin{cases}a_{k} & : u_{k}=0 \\ a_{k}+u_{k} & : u_{k}>0, \text { with probability } 1-\alpha, \\ a_{k}+u_{k}-1 & : u_{k}>0, \text { with probability } \alpha\end{cases}$

this fact is obvious, since the equation does not depend on the service discipline (Bruneel et al. 1993, Laevens et al. 1996a). For 'small' packet-length variances, cases D and C, the FCFS discipline outperforms the RR discipline in terms of mean buffer contents, while for 'large' ones, case $M$, the opposite is true.

Concerning the ATM-switching application, an important observation is that the maximum throughput $\rho_{\max }$, i.e., the maximum number of cells per slot that, on average, can be routed, becomes a function of the switch selection policy. For uniform and homogenous traffic (Awdeh et al. 1995), maximum throughput (Karol et al. 1987) is obtained when

$\lambda \mathrm{E}[d]=\mathrm{E}[u]=1$.

The average packet delay $\mathrm{E}[d]$ (waiting time plus service time) inside a virtual 
queue, equals the average packet service time inside an input queue (Karol et al. 1987). The load of the input queues is thus $\lambda \mathrm{E}[d]$, which should be less than unity. Little's result then yields $\lambda E[d]=E[u]=1$ as stability margin. For a virtual queue using a FCFS scheduling policy on packet level, equations (12) and (13) yield

$\rho_{\max }=\frac{2-\sqrt{2\left(1+V[s] / \mathrm{E}[s]^{2}\right)}}{1-V[s] / \mathrm{E}[s]^{2}}$

as in (Petersen 1991). For virtual queues using a $R R$ discipline on packet level, no closed-form expression could be obtained. However, since the mean buffer contents is nearly insensitive to the exact form of the packet-length distribution, the formula for a geometric distribution (Jacob et al. 1995, $\mathrm{Li}$ 1992, Petersen 1991)

$\rho_{\max }=\mathrm{E}[s](2-\sqrt{4-2 / \mathrm{E}[s]})$

can be used as an approximation. For the four examples studied here, results are summarized in Table 1. Clearly, the packet-length distribution has important consequences for the maximum throughput when the switch uses a FCFS selection policy on packet level.

Table 1 Maximum switch throughput $\rho_{\max }$ (cells/slot)

\begin{tabular}{ccc}
\hline & FCFS & RR \\
\hline D & 0.5858 & 0.5065 \\
\hline C & 0.5445 & 0.5064 \\
\hline G & 0.5064 & 0.5064 \\
\hline$M$ & 0.4772 & 0.5064 \\
\hline
\end{tabular}

\section{(b) Example 2}

The per-type buffer contents is studied by means of a second numerical example. Results are shown in Figures 4 and 5 . We considered a Poisson arrival process whereby, on average, a fraction $\phi$ of the packets has a geometrically distributed length with mean 10.0 , and a fraction $1-\phi$ a geometrically distributed length with mean 20.0. They are referred to in the figures as $G(10.0)$ and $G(20.0)$ respectively. Figure 4 shows the mean per-type and total buffer contents in absolute figures, while Figure 5 shows the mean per-type buffer contents relative to the mean total buffer contents. In the latter figure, 


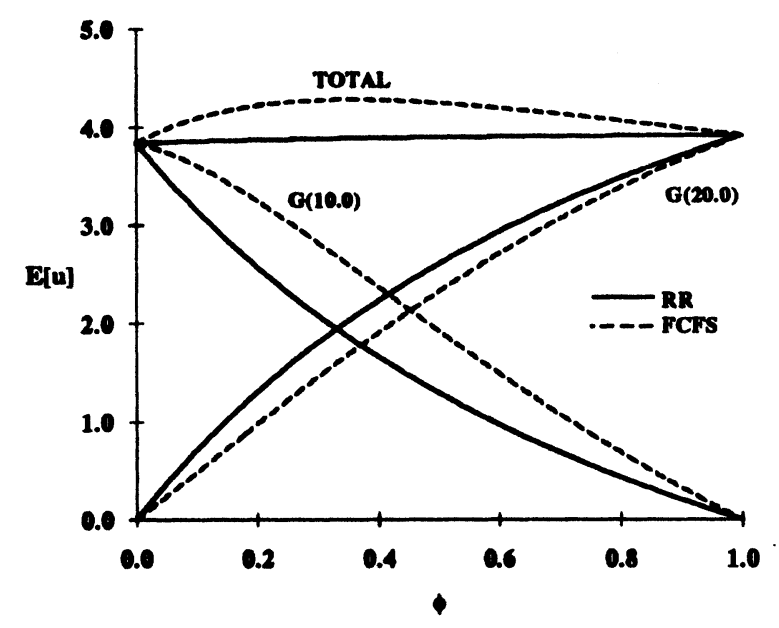

Figure 4 Mean buffer contents per type

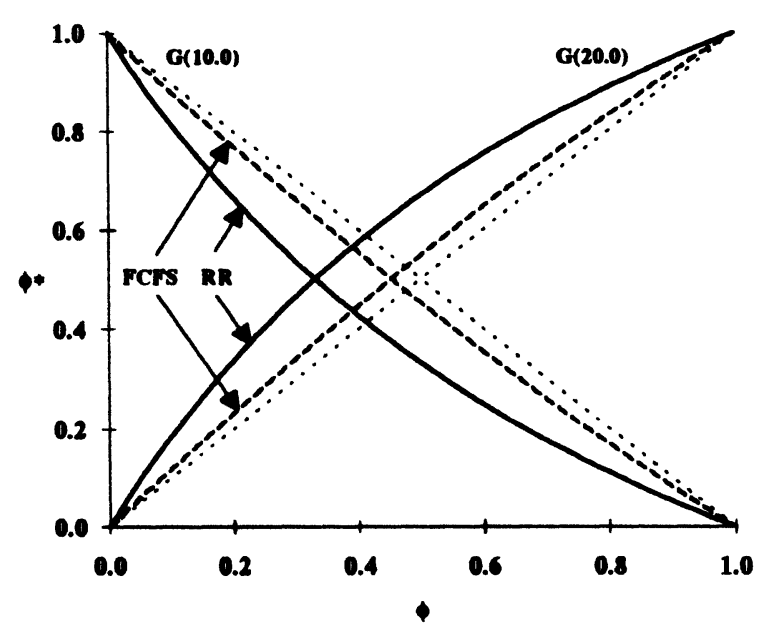

Figure 5 Relative mean buffer contents per type

$\phi^{*}=\mathrm{E}\left[u_{*}\right] / \mathrm{E}[u]$ whereby the mean per-type and total buffer contents relate to the same service discipline, RR or FCFS respectively. The arrival intensity $\lambda$ varies with $\phi$, in order to keep the load constant at $\rho=0.8$. Under the RR discipline, 'shorter' packets, i.e., the ones of type $G(10.0)$, are less abundant than under the FCFS discpline, while the opposite goes for the 'longer' packets. Under the FCFS discipline, the fractions of packet types are nearly the same as those in the arrival stream, shown by the dotted lines in Figure 5. Both fractions would be exactly the same, if one considers only packets in the queue, i.e., the system without the server (Bruneel et al. 1993). The slight 
deviation when considering the buffer contents as in Figure 5, is due to the fact that 'shorter' or 'longer' packets tend to spend, on average, less or more time in the server respectively.

\section{WAITING TIMES}

Concerning waiting times or delays, we can rely on Little's result to calculate mean values, yielding $\mathrm{E}[d]=\lambda^{-1} \mathrm{E}[u]$ and $\mathrm{E}[w]=\mathrm{E}[d]-\mathrm{E}[s]=\lambda^{-1} \mathrm{E}[u]-\mathrm{E}[s]$. In order to obtain, for instance, an expression for the pgf of the waiting time of a tagged packet or for the variance thereof, the analysis in subsection 3.1 of the buffer contents should be modified to some extent. Since the waiting time of a tagged packet is strongly related with the number of other packets in the system during the different cycles it goes through, a joint analysis of these random variables follows closely the derivations made in subsection 3.1 . We do not include this analysis here, but refer the interested reader to (Laevens et al. 1996a), where such an analysis was done for the special case of a geometric packet-length distribution.

In general, we cannot use Little's result to calculate mean packet waiting times conditioned on their initial type, since packets may change type while in the system. We can, however, apply it to a set of types, for which the corresponding states of the underlying Markov chain form a closed subchain, i.e., when they cannot be reached from any of the non-absorbing states outside the set and vice versa. Let us denoted such a set by $H$. Little's result then yields

$\mathrm{E}\left[w \mid \varphi_{1} \in H\right]=\left(\lambda \sum_{i \in H} \nu_{i}\right)^{-1}\left(\sum_{i \in H} \mathrm{E}\left[u_{i}\right]\right)-\mathrm{E}\left[s \mid \varphi_{1} \in H\right]$

This observation provides us with a method to calculate the mean waiting time of a packet, conditioned on its initial state. By defining an alternative Markov chain with, in general, $2 N$ states, in such a way that

$\mathbf{M}^{\prime}=\left[\begin{array}{cc}\mathbf{M} & \mathbf{0} \\ \mathbf{0} & \mathbf{M}\end{array}\right]$

and

$\mathbf{v}^{\prime}=\left(\nu_{1}, \ldots, \nu_{k-1}, 0, \nu_{k+1}, \ldots, \nu_{N}, 0, \ldots, 0, \nu_{k}, 0, \ldots, 0\right)$

we artificially create a closed class for packets whose initial type $\varphi_{1}$ is $k$. Note that both the orginial and new Markov chain correspond nonetheless to a same phase-type distribution. Then, applying the above formula with $H=\{N+1, \ldots, 2 N\}$, we can obtain $\mathrm{E}\left[w \mid \varphi_{1}=k\right]$, after having calculated 
$\mathrm{E}\left[u_{i}\right]$ for $i=N+1, \ldots, 2 N$. In general, this approach doubles the number of states in the Markov chain, and hence quadruples the matrix involved in solving equation (11). Moreover, it should be repeated for every conditional mean waiting time one wants to obtain.

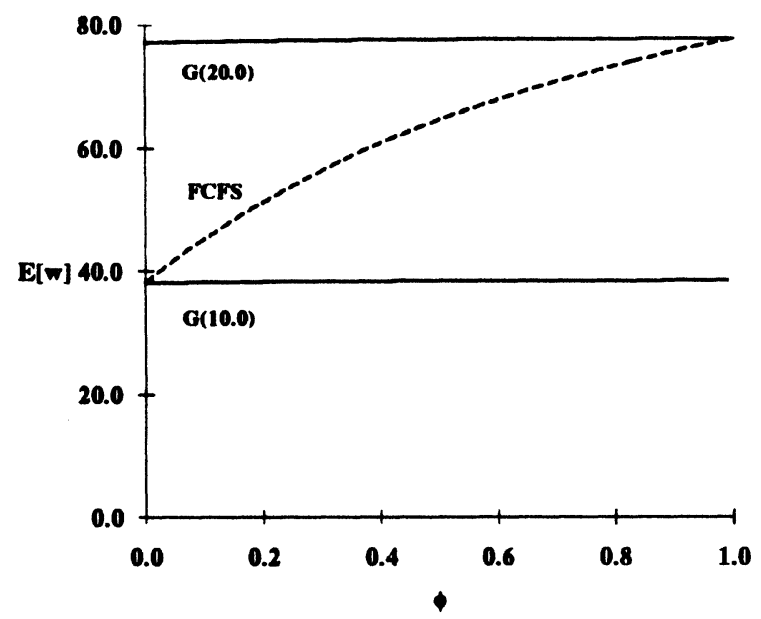

Figure 6 Mean waiting time per type

We conclude this section with Figure 6, that complements Figures 4 and 5. Shown is the mean waiting time under the RR discipline of packets with geometrically distributed length with mean 10.0 and 20.0, denoted $G(10.0)$ and $G(20.0)$ respectively, that mix in the arrival process in fractions $\phi$ and $1-\phi$, as in numerical example 2. Clearly, under the RR discipline the mean waiting time of a packet of one type is only slightly disturbed by the presence of packets of another type. For comparison, the mean waiting time under the FCFS discipline, which is the same for all types of packets, is also shown. Under this discipline 'shorter' packets suffer from the presence of 'longer' packets.

\section{CONCLUSIONS}

A system of functional equations was derived for multivariate generating functions related to the queue contents in a discrete-time single-server queue, based on a kind of memoryless property of the phase-type distribution that was assumed for the packet lengths. From this complex system of equations, a practical method to calculate the mean buffer contents was obtained. Little's result then yielded mean waiting times. Calculation of higher-order moments of the buffer contents, such as variances, is just a matter of performing some straightforward algebra, along the lines of subsection 3.2. A topic for future re- 
search certainly is the question whether tail probabilities can also be extracted from the functional equations, be it through numerical transform inversion or by an approximate analytical approach.

Comparing the RR discipline with the FCFS discipline showed that the former does not always perform optimal in terms of mean buffer contents, or, within the context of ATM-switching, in terms of maximum throughput. However, since it is quasi insensitive to the exact form of the packet-length distribution, a switch design implementing the RR discipline would be more robust. Besides this, the discipline also has the advantageous feature of being fair, in the sense that it relates packet waiting-times inside the virtual queues to packet lengths.

To keep focus on the main ideas of the proposed approach, the analysis was restricted to the essentials. We hope a more detailed analysis, incorporating e.g. higher-order moments of the waiting time, may become the subject of future reports.

\section{REFERENCES}

Asmussen, 0., Nerman, 0. and Olsson, M. (1996) Fitting phase-type distributions via the EM algorithm. Scandinavian Journal of Statistics, 23, 419-441.

Awdeh, R. and Mouftah, M. (1995) Survey of ATM switch architectures. Computer Networks and ISDN Systems, 27, 1567-1613.

Bruneel, H. and Kim, B. (1993) Discrete-time models for communication systems including ATM. Kluwer Academic Publishers, Boston.

Cao, X.-R. (1995) The maximum throughput of a nonblocking space-division packet switch with correlated destinations. IEEE Transactions on Communications, 43, 1898-1901.

Cao, X.-R. and Towsley, D. (1995) A performance model for ATM switches with general packet length distributions. IEEE/ACM Transactions on Networking, 3, 299309.

Daduna, H. and Schassberger, R. (1981) A discrete-time round-robin queue with Bernoulli input and general arithmetic service time distributions. Acta Informatica, 15, 251-263.

Disney, R.L., Konig, D. and Schmidt, V. (1984) Stationary queue-length and waiting time distributions in single server feedback queues. Advances in Applied Probability, 16, 437-446.

Jacob, L. and Kumar, A. (1995) Saturation throughput analysis of an input queueing ATM switch with multiclass bursty traffic. IEEE Transactions on Communications, 43, 757-761

Jaiswal, N.K. (1982) Performance evaluation studies for time-sharing computer systems. Performance Evaluation, 2, 223-236.

Karol, M.J., Hluchyj, M.G. and Morgan, S.P. (1987) Input versus output queueing on a space-division packet switch. IEEE Transactions on 
Communications, 35, 1347-1356.

Kleinrock, L. (1976) Queueing systems, volume II: computer applications. Wiley, New York.

Laevens, K. and Bruneel, H. (1996a) Discrete-time queueing models with feedback for input-buffered ATM switches. Performance Evaluation, 27\&28, 71-87.

Laevens, K. and Bruneel, H. (1996b) Useful relations in the analysis of ATMqueues fed by multiple types of traffic. Electronics Letters, 32, 631-632.

Lam, S.S. and Shankar, A.U. (1981) A derivation of response time distributions for a multi-class feedback queueing system. Performance Evaluation, 1, 48-61.

Li, S.-Q. (1992) Performance of a nonblocking space-division packet switch with correlated input traffic. IEEE Transactions on Communications, 40, 97-108.

Muntz, R. (1972) Waiting time distributions for round-robin queueing systems. Proceedings Symposium on Computer and Communications Networks and Teletraffic (Brooklyn, 1972)

Neuts, M.F. (1981) Matrix-geometric solutions in stochastic models. John Hopkins University Press, Baltimore.

Petersen, J. (1991) Throughput limitation by head-of-line blocking. Proceedings ITC-13 (Copenhagen, 1991), 659-663.

Schassberger, R. (1981) On the response time in a discrete round-robin queue. Acta Informatica, 16, 57-62.

Schassberger, R. (1984) A new approach to the M/G/1 processor-sharing queue. Advances in Applied Probability, 16, 202-213.

Takács, L. (1963) A single-server queue with feedback. The Bell System Technical Journal, 42, 505-519.

van den Berg, J.L., Boxma, O.J. and Groenendijk, W.P. (1989) Sojourn times in the $\mathrm{M} / \mathrm{G} / 1$ queue with deterministic feedback. Stochastic Models, 5, 115-129.

van den Berg, J.L. and Boxma, O.J. (1991) The M/G/1 queue with processor sharing and its relation to a feedback queue. Queueing Systems, 9, 365-40.

\section{BIOGRAPHY}

K.Laevens obtained his Masters Degree in Electrical Engineering at the University of Ghent, Belgium, in 1991. He then joined the SMACS Research Group (Stochastic Modeling and Analysis of Communication Systems) at that university, where he is currently working towards a $\mathrm{Ph} . \mathrm{D}$. Degree. Besides the theoretical aspects of (discrete-time) queueing theory, his research interests also include the performance evaluation of communication systems - in specific components of the ATM network - and traffic characterization and modeling. 